The $B D J$ News section accepts items that include general news, latest research and diary events that interest our readers. Press releases or articles may be edited, and should include a colour photograph if possible. Please direct your correspondence to the News Editor, Arveen Bajaj at the BDJ, The Macmillan Building, 4 Crinan Street, London N1 9XW or by email to bdj@bda.org

\section{Children's oral health reflects disparities}

Researchers from eight countries have reported that, while some progress has been made in improving the oral health of children and adolescents, much remains to be done. Presenting their findings during the 84th General Session of the International Association for Dental Research, research teams found that targeted health promotion and increased access to dental services are needed to improve the oral health of adolescents in juvenile detention (Australia). A study at the Shanghai Children's Medical Center raised awareness of early childhood caries, the prevalence of which is extremely high (China) while Japanese researchers found that there was significant differences among geographical regions in terms of caries prevalence in deciduous teeth, and the factors associated with dental caries prevalence.

Finnish researchers found that the health system, oral health services, and socio-cultural and environmental risk factors (e.g., the use of alcohol and tobacco products) did modify, to some extent, the effects of risk factors such as infrequent use of oral health services, and unhealthy habits, in teenagers and an Irish pilot questionnaire, the Ado-

lescent Enamel
Fluorosis Quality
of Life Question-
naire, indicated
the aesthetic
impact of enamel
fluorosis in 15-
year-olds, and its
impact on their
oral-health-relat-
ed quality of life

\section{Dundee Dental Education Centre Opens}

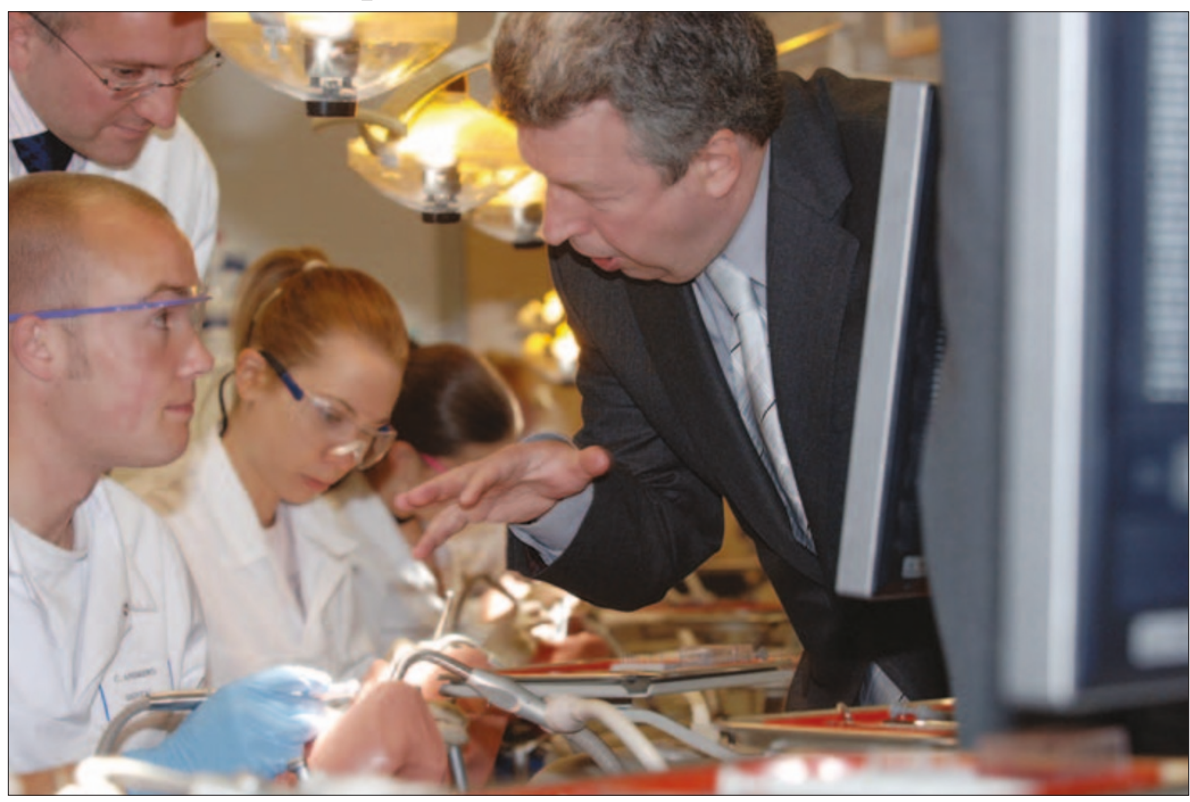

A £3 million state-of-the-art dental facility has recently been opened in Dundee. The Dundee Dental Education Centre brings together NHS Tayside, NHS Education for Scotland (NES) and the University of Dundee, and provides a flagship facility and a focus for education and training for dentistry in the east of Scotland. The Centre has been created within the Frankland Building on the University of Dundee campus, which is now joined by a physical link to the neighbouring Dundee Dental Hospital and School.

The facility will provide postgraduate dental education and clinical skills training for all dental professionals and will also support pre-registration training for dental nurses and undergraduate education.

The most striking feature in the new centre is a "phantom heads" clinical skills area, providing a stunning educational facility for both postgraduate and undergraduate training. This unit comprises 38 fixed student simulators with phantom heads and fully integrated audiovisual facilities, allowing advanced hands-on training.

Lewis Macdonald, Deputy Minister for Health and Community Care, opened the Centre and commented, "The centre will play a crucial role in the training and development of the next generation of NHS dentists as well as other members of the dental team. This new centre also has an important role to play in the outreach training programme for students which will see students from Dundee Dental School training in communities across the North and North East. The outreach training programme should encourage dentists to remain within these communities to practice once they have completed their training."

Mrs Ann Markham, OBE, Chair of NHS Education for Scotland said, "Dentists and dental care professionals will benefit from training which is based on the quality evidence-based guidelines which are being produced by the Dental Clinical Effectiveness Group based at the centre. Tutors there will use these guidelines, along with sound educational principles, to deliver training to an expanding client base. Of particular interest is the training provided for the Polish dentists who are being recruited to the Salaried Dental Service in Scotland, as well as introductory courses for other overseas dentists who wish to work in the NHS." 
DIARY

September

The British Society of Paediatric Dentistry

Annual Scientific Conference

'The Art \& the Science'

Venue: The Hilton, Leeds City

Date: 12-15 September 2006

www.bspd.co.uk/conf-2006.html

Moving to private practice

Venue: The Midland Hotel, Manchester

Date: 8 September 2006

www.bda-events.org

European Society for Oral Laser Applications/ Hellenic Society for Oral Laser Applications 1st Mediterranean Laser Congress

Venue: Hilton Hotel Rhodes Resort, Greece

Date: 21-23 September 2006

Email: esola2006@medacad.org

www.esola.at

FDI Annual World Dental Congress

Venue: Shenzhen, China

Date: 22-25 September 2006

Email: congress@fdiworldental.org

www.fdiworldental.org

October

The American Dental Association (ADA) 147th ADA Annual Scientific Session and

Marketplace Exhibition

Venue: Mandalay Bay Resort and

Convention Center, Las Vegas

Date: 16-19 October 2006

www.ada.org

Preparing for retirement

Venue: Cedar Court Hotel, Harrogate

Date: 6 October 2006

www.bda-events.org

Community Dental Services Group Annual

Presidential and Scientific meeting

Venue: Marriott Forest of Arden Hotel,

Birmingham

Date: 12-13 October 2006

www.bda-events.org

Hospitals Group Annual Presidential and

Scientific meeting

Venue: Portmeirion, North Wales

Date: 26-27 October 2006

www.bda-events.or

November

British Academy of Cosmetic Dentistry's (BACD) 2nd Annual Conference

Venue: Britannia International Hotel, London

Date: 16-17-18 November 2006

Email:info@bacd.com

www.bacd.com

\section{Cranberries can combat caries}

Scientists have discovered that the humble cranberry harbours several anti-oxidants (flavonoids) that show the ability to counteract the damaging effects of the bacterium streptococcus mutans, which causes dental caries.

The researchers from the University of Rochester in New York, found that cranberries harbour a plethora of biological compounds such as flavonoids (e.g., quercetin and myricetin), phenolic acids (benzoic acid), anthocyanins, and condensed tannins, among others. They found that many of these substances could inhibit enzymes associated with the formation of the plaque polysaccharide matrix, block the adherence of bacteria to surfaces, prevent acid formation, and reduce acid tolerance of cariogenic organisms.

The next step in their research is to identify the specific active constituents in cranberries that could be useful as anti-caries/anti-plaque agents.

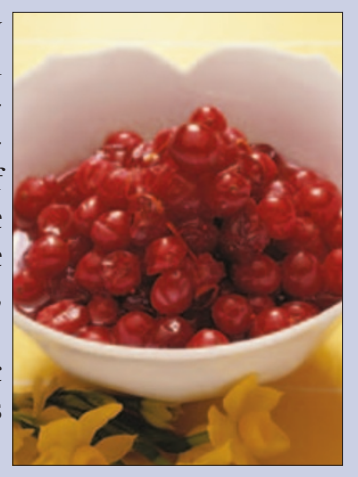

\section{Double award winner}

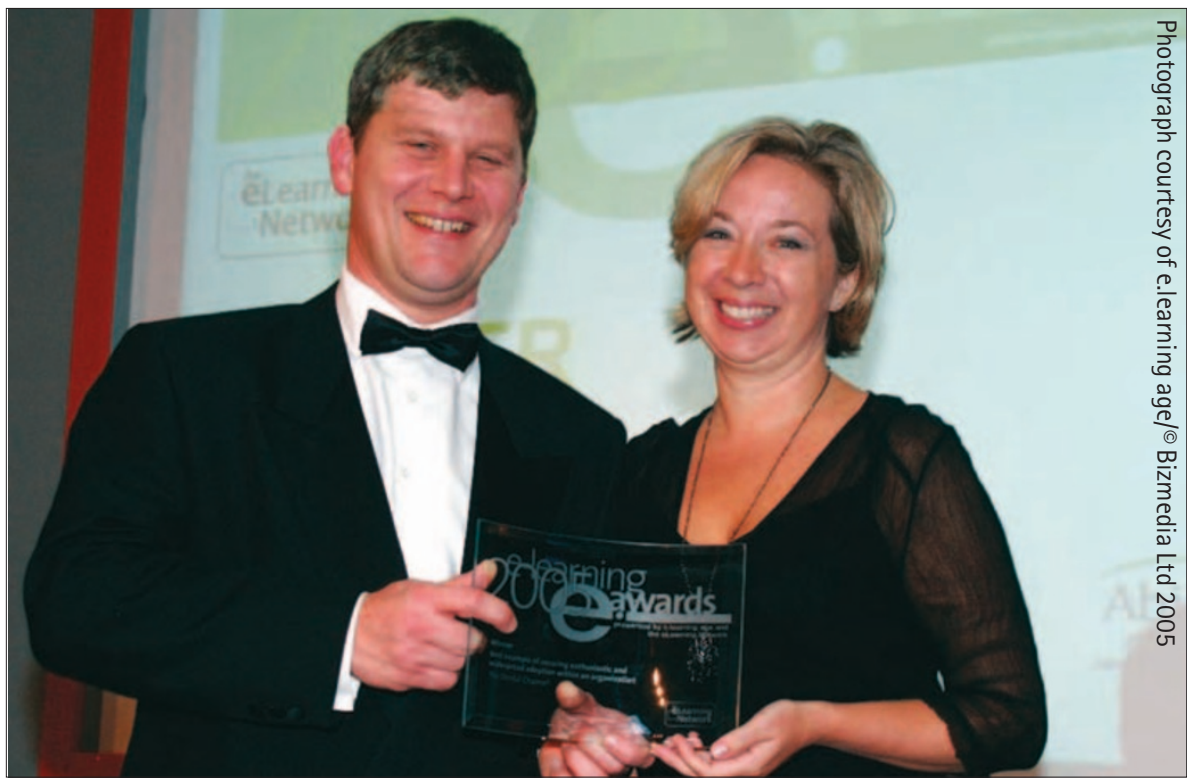

An interactive guide to endodontic best practice on CD-ROM has won two major independent industry awards. Pulp Afflictions, the Dental Channel's peer-reviewed interactive guide has won the AV Awards Digital Media Project of the Year 2005 and the E-learning Awards 2005 Project or strategy that has done the most to secure enthusiastic and widespread adoption of e-learning within an organisation.

The project's success was attributed to factors including its incorporation of a range of techniques (e.g hypertext, interactive images, video etc) and comprehensive peer-review.

\section{Dental disease and risk of stroke study}

Researchers have discovered an association between gum disease and the increased risk of stroke due to potential blockages of the main blood vessels leading to the brain (carotid artery).

These blockages, or atheromas, contain calcium and can be detected on dental panoramic radiographs. Given this finding, Drs E. Chung, A.F. Friedlander, E.C. Sung, and N.R. Garrett, from the University of California-Los Angeles, USA, conducted a study to determine if dental disease depicted on a panoramic radiograph is more prevalent among people with atheromas seen on their dental radiograph than among people without atheromas, but matched for stroke risk factors (body mass, smoking history, need for medications to control hypertension, hypercholesterolemia, and diabetes). The group with carotid atheromas seen on dental panoramic radiographs was found to have more dental disease, as determined by the number of teeth with decay, missing teeth, and the amount of bone loss around teeth, than the group without any radiographically detectable atheromas. Researchers claim that the results of this study indicate that dental disease may play a role in the formation of carotid atheromas in patients already at risk for stroke. 


\section{Tooth tissue engineering}

It could be possible to generate bioengineered dental tissues, according to research from The Forsyth Institute (Boston, MA, USA) presented at the 84th General Session of the International Association for Dental Research. The team found that mixed populations of cultured post-natal tooth bud cells can be used to generate bioengineered dental tissues.

Current research efforts focus on the identification and characterisation of dental cell populations, scaffold materials, and design that can be most effectively used for tooth tissue engineering applications.

Hoechst dye profiling and immuno-sorting methods were used to generate enriched clonal dental stem cell (DSC) lines. Expanded DSC and non-DSC lines are currently being examined, by both in vitro and in vivo methods, to define their potential to differentiate. The researchers say that molecular and differentiation profiles will provide important characterisations of tooth bud cells, to eventually facilitate ongoing tooth tissue engineering efforts.

\section{Steroid abuse can harm gingival tissues}

Researchers from Cukurova University in Turkey have found that prolonged use of anabolic androgenic steroid (AAS) is closely associated with significant levels of gingival enlargement, according to their new study published in the Journal of Periodontology.

The researchers found that AAS abusers had statistically significant levels of gingival enlargement compared to non-users, requiring a gingivectomy for many cases. They examined 24 athletes between the ages of 17 and 29 who had been using AAS for more than one year. All subjects were examined for plaque levels, gingival inflammation and gingival enlargement. The results were then compared with a control group of 20 bodybuilders who had never used AAS drugs and matched for age, educational level and oral habits according to the data obtained from the AAS user group.

Onur Ozcelik, DDS, PhD, Faculty of Dentistry, Department of Periodontology, Cukurova University, Adana, Turkey said, “Although it has been reported that many of the adverse effects of AAS abuse are fully reversible within several months after the cessation of the drug, it is not known if gingival enlargement would also regress after the withdrawal of AAS."

Researchers also found that gingival inflammation was higher in the AAS user group compared to the non-AAS users. "Further studies are required to find out if increased gingival scores in the user group are a direct effect of AAS or if the inflammation is a result of compromised oral hygiene due to gingival enlargement," said Ozcelik.

He suggested that people taking AAS without medical supervision should be informed of the adverse effects and strongly encouraged to begin a cessation program.

\section{GDC celebrates $50^{\text {th }}$ anniversary}

The General Dental Council (GDC) has celebrated its golden anniversary which marked 50 years since the GDC's first meeting on 4 July 1956 when Sir Wilfred Fish was elected President.

To mark the occasion, Dame Janet Smith delivered the biennial Wilfred Fish lecture on the subject of 'Where now for the regulators?'. In her speech Dame Janet spoke about regulation following the Shipman case and other medical scandals. In addition she reviewed the objectives of regulatory bodies and suggested functions for all regulators.

Commenting on the 50th anniversary GDC President, Hew Mathewson, said, "I am delighted to be the President of the GDC in its golden anniversary year. Those 50 years have seen an astonishing amount of change, not simply in the work of the average dentist but also in the size of the team as a whole and in the roles of the team. This summer's opening of a statutory register for dental nurses, technicians, and other dental care professionals will herald a period of further change, including the addition of an estimated 40,000 registered professionals to the workforce. I am confident that the practice of dentistry in the UK will continue to develop in a vibrant and forward looking way." The Wilfred Fish lectures take place every two years and were founded in honour of the first President of the GDC, Sir Wilfred Fish, who was elected to the Dental Board of the United Kingdom in 1939.

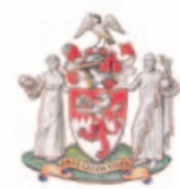

General Dental Council 


\section{Effects of dental amalgam fillings in children}

A new study has been conducted into the effects of placing amalgam fillings, which contain mercury, into children's teeth.

Both studies - one conducted in Europe, the other in the United States - independently reached the conclusion that children whose cavities were filled with dental amalgam had no adverse health effects.

The findings included no detectable loss of intelligence, memory, coordination, concentration, nerve conduction, or kidney function during the 5-7 years the children were followed. The researchers looked for measurable signs of damage to the brain and kidneys, because previous studies with adults indicated that these organs might be especially sensitive to mercury.

The authors noted that children in both studies who received amalgam had slightly elevated levels of mercury in their urine. But after several years of analysis, they determined that the mercury levels remained low and did not correlate with any symptoms of mercury poisoning.

The two studies were the New England study, which was undertaken in the urban Boston (MA) area and rural Farmington (Maine), and the Portuguese study, conducted in Lisbon, Portugal. Each study enrolled over 500 children who had existing untreated decay in permanent posterior, or back, teeth, but no previously placed dental amalgam fillings. Each child was randomly assigned to receive

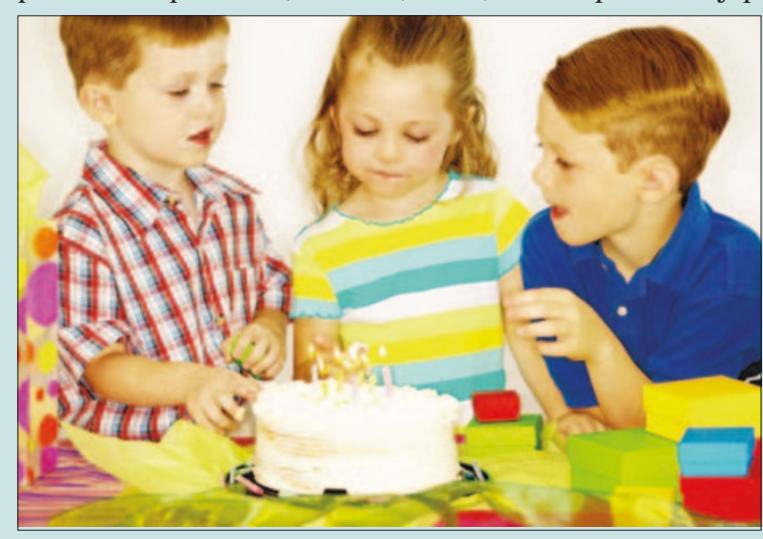
either amalgam or composite resin (tooth-colored) fillings while participating in the research studies. All were evaluated for several years thereafter to determine if any health changes occurred, with emphases on IQ changes in the New England study and on memory, concentration, coordination, and nerve conduction measures in the Portuguese study.

The scientists noted that children in both groups were in great need of dental care. Among those in the amalgam group, children had, on average, 10.1 tooth surfaces treated upon entry into the study. By year seven of the study, they had received, on average, a total of 18.7 surface restorations. Each tooth has either four or five defined surface areas, totaling 128 surfaces in the 32 permanent teeth.

The findings were presented during the 84th General Session of the International Association for Dental Research, convening at the Brisbane Convention \&t Exhibition Centre.

\section{Water fluoridation is cost-effective}

Teams of investigators from the University of Melbourne and New South Wales Health have reported the results of a project investigating the impact of changing dental needs on the cost savings from community water fluoridation in Melbourne, Australia.

They were able to track how cost savings from water fluoridation vary over time for any given age group and level of dental decay. Central to the project was their ability to take into consideration the effects of an aging population, lower rates of edentulism, and consequent higher rates of treatment need for the gums and supporting tissues of the teeth. The findings suggest that, even in an era of declining levels of dental decay and supplementary sources of fluoride, water fluoridation continues to be a cost-effective preventive measure, with annual per person savings ranging from just over AUS\$56 in the 1970 s to just under AUS\$18 in the 1990s. At each of the time points analysed, however, the cost savings from water fluoridation were shown to decline with age, largely as a result of a plateau in the amount of new tooth decay experienced, lower averted costs of decayed surfaces experienced later in life and estimates of higher periodontal treatment needs compared with those of younger age groups.

The authors of the report, Impact of Changing Dental Needs on Cost Savings from Fluoridation, by A.C. Campain, M.V. Morgan, R.J. Marino, and C. Wright say the research lends strong support to the continued application of community water fluori-

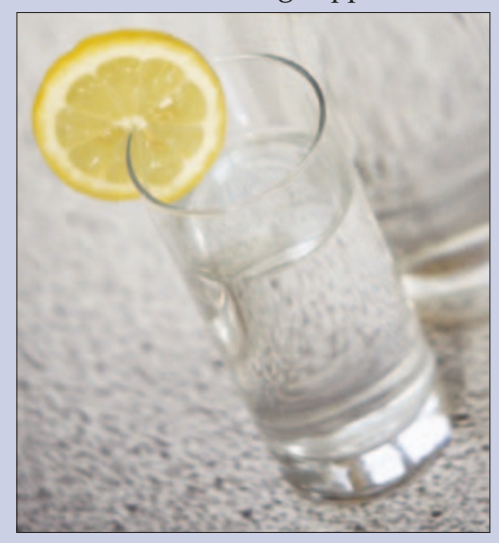

dation as an efficacious and cost-saving public health measure.

However, as the trend toward greater tooth retention in an aging population continues, the prospect that the cost-saving benefits of water fluoridation might be offset to some degree by the potentially high costs of periodontal treatment needs later in life and highlights the need for public dental health professionals and policymakers to begin to give consideration to community-based strategies which will be effective in containing these costs. The report was presented at the 84th General Session of the International Association for Dental Research.

\section{Reducing alveolar bone loss}

Heat-shock protein (HSP) can be used as a vaccine to cross-protect against multiple pathogenic species, according to investigators from Pusan National University (South Korea). They presented the findings of a study they performed to evaluate the bacterium Porphyromonas gingivalis heatshock protein (HSP) 60 as a vaccine candidate to inhibit multiple bacteria-induced alveolar bone loss.

Recombinant P.gingivalis HSP60 was produced and purified from P.gingivalis GroEL gene. Rats were immunized with P.gingivalis HSP60, and experimental alveolar bone loss was induced by infection with multiple periodontopathogenic bacteria.

The researchers found that there was a very strong inverse relationship between post-immune anti-P.gingivalis HSP immunoglobulin $G$ levels and the amount of alveolar bone loss induced by either P.gingivalis or multiple bacterial infection $(p=0.007)$. Analysis of data from polymerase chain-reaction indicated that the vaccine successfully eradicated the multiple pathogenic species. They concluded that P.gingivalis HSP60 could potentially be developed as a vaccine to inhibit periodontal disease induced by multiple pathogenic bacteria. 


\section{New method of jaw reconstruction for oral cancer patients}

Surgeons from the Mayo Clinic (USA) claim they have developed a promising new process for mandible reconstruction following removal of oral cancer.

The new method, which the investigators tested in rabbits, used distraction osteogenesis after tumor removal surgery to restore the missing portion of a patient's jaw. With distraction osteogenesis, the surgeon made a cut at one of the remaining ends of the patient's jawbone. As the break healed, the first phase of growth, soft tissue, appeared. The surgeon then stretched the pliable soft tissue under the tension of a device to fill in the gap in the patient's jaw. Within 24 hours of completing the stretching process, physicians started administering radiation therapy to the mandible to decrease the likelihood of cancer recurrence. As the soft tissue healed, it hardened into bone. The investigators found that the radiation therapy, which they had suspected could interrupt the bone consolidation prior to the study, had no ill effects on the bone's healing.

"Patients with large oral tumors should have radiation therapy after surgery to decrease the chances of cancer recurrence," says Dr Daniel Price, M.D., Mayo Clinic ear, nose and throat surgery resident and lead study investigator. "Radiation needs to be completed 13 to 14 weeks after the patient's cancer diagnosis to achieve maximum effect. As these patients require mandible reconstruction after tumor removal, we wanted to find a way to get both the radiation and the reconstruction done quickly and concurrently."

Patients who might be candidates for the new reconstructive and treatment procedure have oral cancer involving part of the mandible. To completely remove the tumor surgically, surgeons have to remove part of the mandible. Without reconstructive surgery, patients would have difficulty eating and speaking, and would develop a significant facial deformity, says Eric Moore, M.D., Mayo Clinic ear, nose and throat surgeon and senior study investigator.

Dr. Price says that it is also preferable to start the reconstruction when the tumor is removed, as demonstrated in this method, rather than wait until radiation therapy is completed, because immediate reconstruction minimises scar tissue and is better tolerated by the patient. The standard procedure for jaw reconstruction following removal of an oral cancer is transfer of bone from the patient's fibula in the leg, along with surrounding muscle, skin and the supplying vessels. Although the aesthetic and functional result for the mandible can be good, this procedure has drawbacks.

"If we could avoid the whole process of going to another part of the body in order to reconstruct a patient's jaw, it would be best," says Dr. Moore. Drs Price and Moore had been performing similar distraction osteogenesis in children born with small jawbones and wondered about the feasibility of using the procedure for adult oral cancer patients. They embarked on this research project to test it in animals and have tried this method on human oral cancer patients who were not appropriate candidates for the fibula transfer surgery. They have also utilized the method in patients who have benign tumors or who have suffered a trauma.

The patients who have had distraction osteogenesis performed by Drs. Price and Moore compared the level of discomfort to having orthodontic braces. The devices used for the stretching procedure are submerged and not visible.

The next step in this research, according to Dr. Price, is to study the distraction osteogenesis plus radiation therapy method in larger animals, comparing them to animals who are given distraction osteogenesis without radiation therapy.

Details of the study were presented in June at the International Federation of Head and Neck Oncologic Societies meeting in Prague, Czech Republic.

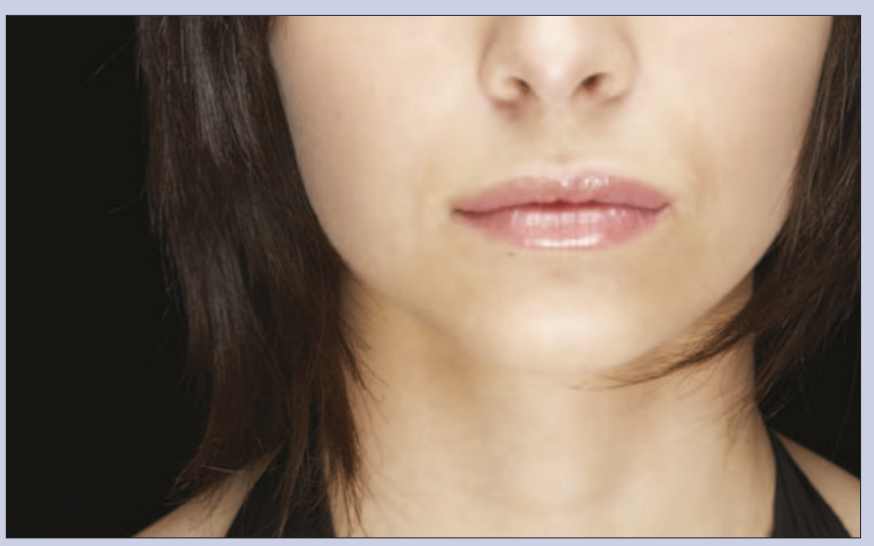

\section{Periodontal disease and health care costs}

Prevention of periodontal disease may lead to saving of not only dental care but also medical care costs, according to a report by researchers at the Institute of Industrial Ecological Sciences, University of Occupational and Environmental Health, Kitakyushu,

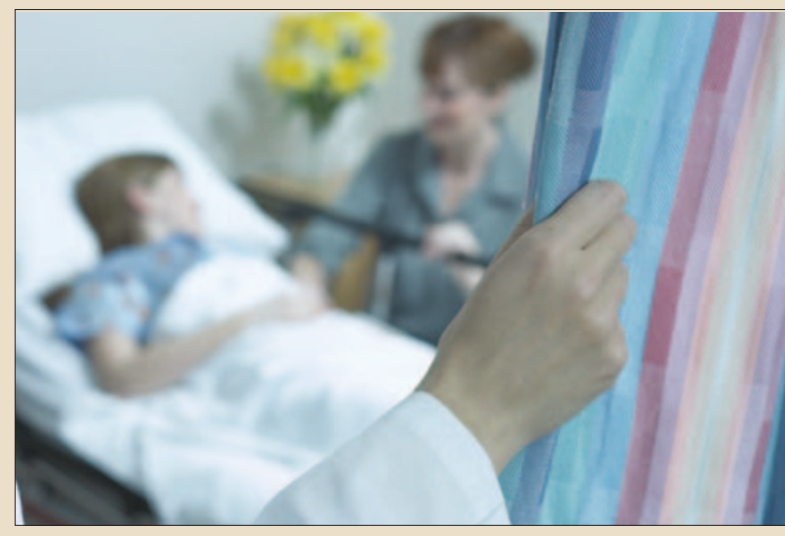
Japan. Researchers evaluated the effects of periodontal disease on the use and costs of medical and dental care using data from worksite health and dental examinations and health insurance claims of 4,285 civil officers aged 40-59 years. Those with severe periodontitis accrued 21 per cent higher total costs than those with no pathological pocket (periodontal pocket probing depth less than $4 \mathrm{~mm}$ ) over the 3.5-year period. The admission rates of those with severe periodontitis were high for both sexes. In males, the dental costs for this group were approximately twofold higher than those with no pathological pockets

The investigators, R. Ide, T. Hoshuyama, and K. Takahashi, presented their research at the 84th General Session of the International Association for Dental Research and concluded that periodontal disease had a significant impact on health care cost increases, through not only dental care costs but also inpatient care costs, especially in males. 